\title{
Análisis
}

\section{“365 días de libros": blog para la promoción de la lectura}

Por Francisco López Hernández y Honorio Penadés de la Cruz

Resumen: Presentación de un blog institucional, creado en la biblioteca del campus de Colmenarejo de la Universidad Carlos III de Madrid, dedicado a la promoción de la lectura entre universitarios. Tras unas reflexiones sobre las posibilidades de un medio digital participativo para incentivar el uso de las colecciones de literatura de la biblioteca se describen los pasos dados por ésta en una campaña que incluye adquisiciones, exposiciones, y reseñas bibliográficas en el blog.

Palabras clave: Promoción de la lectura, Blogs, Bibliotecas universitarias.

Title: "365 days of books": a blog to promote reading

Abstract: Description of an institutional blog, created by the library of the Colmenarejo campus of the University Carlos III of Madrid. The aim of the blog is to promote reading among the university population. After first assessing the potential of this participative digital medium for encouraging the use of literature collections, the

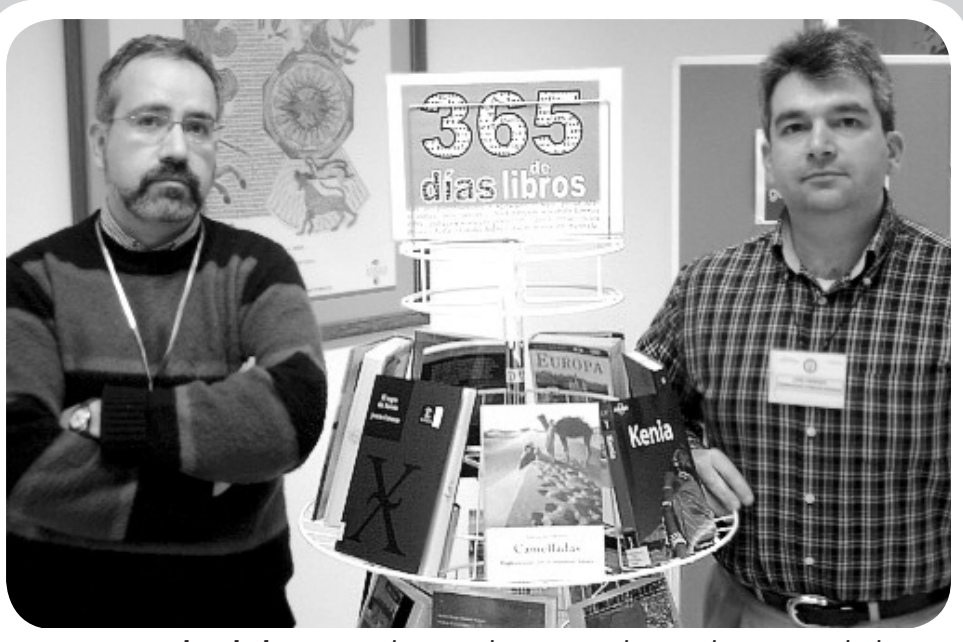

Honorio Penadés de la Cruz es licenciado en Derecho por la Universidad Complutense de Madrid y bibliotecario en la Universidad Carlos III de Madrid desde 1993; actualmente coordina los mostradores de la Biblioteca del campus de Colmenarejo.

Francisco López Hernández es licenciado en farmacia por la Universidad Complutense de Madrid y bibliotecario en la Universidad Carlos III de Madrid desde 1993; actualmente es jefe de sección de Atención a Usuarios en la biblioteca del campus de Colmenarejo.

article describes the steps taken by the library in this campaign that include acquisitions, exhibitions and book reviews posted to the blog.

Keywords: Reading promotion, Weblogs, Blogs, Academic libraries.

López Hernández, Francisco; Penadés de la Cruz, Honorio. "365 días de libros: blog para la promoción de la lectura". En: El profesional de la información, 2007, v. 16, n. 2, pp. 131-133.

DOI: 10.3145/epi.2007.mar.05

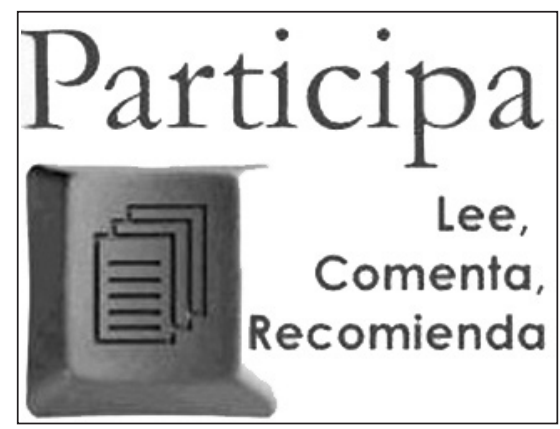

Introducción

PRESENTAMOS UN CASO DE INTEGRACIÓN de una de las más tradicionales funciones de los bibliotecarios (recomen- daciones de lectura) con una de las herramientas más pujantes en la actualidad (los blogs institucionales). Está realizado con el objetivo de acercarnos a una

\section{"Un blog con reseñas} de libros mejoraría la visibilidad y aumentaría el uso de las colecciones para el ocio, apoyando las exposiciones bibliográficas" de las características fundamentales de la web 2.0 como es la participación. En este caso de los lectores y en un entorno (una biblioteca universitaria) que en principio no tiene entre sus misiones destacadas la promoción de la lectura.

Se trata de un blog bibliotecario llamado 365 días de libros, donde se comenta periódicamente una selección de "lecturas para el ocio" que complementa las colecciones académicas de la biblioteca del campus de Colmenarejo de la Universidad Carlos III de Madrid. 


\section{"Pensamos que un blog convertiría a los alumnos universitarios en amantes de la lectura y a los bibliotecarios en críticos literarios, o viceversa"}

\section{http://365diasdelibros.blogspot.com}

En él participan Honorio Penadés como administrador y Rocío Botías como encargada de las adquisiciones, pero también cualquier miembro del personal de la biblioteca que desee aportar sus conocimientos realizando recensiones o recomendaciones. Desde su puesta en marcha, y durante los primeros seis meses de funcionamiento, ha recibido más de 9.000 visitas y se han reseñado 26 títulos de libros.

No vamos a entrar a estas alturas a definir un blog ni a elucubrar sobre su tipología o su carácter participativo, pero vamos a destacar ciertas características que nos llevaron a usarlo: es la evolución del tablón de anuncios, del boletín de novedades, de la charla de amigos y de aquel "what's new, what's cool" de las webs de los noventa. Es un cuaderno de navegación, pero lo es también de ideas según la clásica definición de Merholz, "una especie de autobiografía intelectual muy informal y extemporánea, escrita con la doble intención de provocar el diálogo y servir de depósito para el pensamiento" (Candeira). La Red se infla como una "burbuja intelectual" gracias a los recursos de participación individual, desde El rincón del vago a los repositorios institucionales open access, la Wikipedia o los blogs.

Por otra parte, si el trabajo medio de un bibliotecario tiene un 50\% de rutina (presta, devuelve, selecciona, compra, cataloga, informa, etc.), tiene otro porcentaje similar de creatividad (programa, innova, busca, reorganiza, mejora, etc.) y la oportunidad de usar un recurso que ha transformado la tradicional estructura de los medios de comunicación, nos permitiría convertir a los alumnos universitarios en amantes de la lectura y a los bibliotecarios en críticos literarios, o viceversa. Entra aquí otro concepto muy de moda, el de la inteligencia emocional: "Hoy en día lo que cuenta es el capital intelectual. Los trabajos rutinarios pueden ser realizados por las máquinas, de modo que el trabajo humano se centra en la creatividad, el compromiso y las habilidades sociales, entre las que se incluye la inteligencia emocional. La tecnología facilita la posibilidad de realizar el trabajo donde se quiera y cuando se quiera, de modo que damos la bienvenida al trabajo sin fisuras, sin principio ni fin" (Berndton).

\section{Un poco de historia}

Desde otoño de 2004 la biblioteca venía realizando pequeñas exposiciones bibliográficas en su vestíbulo de entrada con ocasión de ciertos eventos que tenían lugar en el campus o con relación a determinadas fechas destacables (el día del libro, por ejemplo). Desde mayo de 2005 se procuró potenciar la colección de narrativa ("lecturas para el ocio") mediante la adquisición de libros de los géneros más demandados. Se planeó un calendario de exposiciones monográficas según los géneros literarios y se compró un "carrusel", un mueble expositor de libros que funciona como un escaparate. Es semejante a un expositor de supermercado e invita a hojear y llevarse el ejemplar. Es entonces cuando, para mejorar aún más la visibilidad de la colección, se pensó un sistema de difusión más extenso: un blog.

\section{Próximos temas centrales} Mayo 2007 Portales y gestión de contenidos (CMS) Julio 2007 Bibliotecas universitarias Septiembre 2007 Documentación multimedia Noviembre 2007 Ontologías

\section{Enero 2008 Software libre para bibliotecas}

Los interesados pueden remitir notas, artículos, propuestas, publicidad, comentarios, etc., sobre estos temas a: epi@elprofesionaldelainformacion.com 


\section{DOls en EPI}

A partir de este ejemplar, todos los artículos y notas de EPI cuentan con el document identification number. CrossRef, la organización que gestiona los dois por encargo de Pila (Publishers International Linking Association, Inc) ha asignado a EPI el número de editorial 10.3145/

CrossRef mantiene una base de datos actualizada de manera que buscando o haciendo click sobre un doi, siempre se encuentra el artículo correspondiente.

\section{EPI irá asignando dois retrospectivamente a todos sus artículos hasta el año 2000. http://www.crossref.org}

\section{¿Por qué un blog?}

Es un medio profusamente utilizado por los jóvenes (Celaya), un $84 \%$ de los cuales navega habitualmente por internet, el $53 \%$ utiliza la Red para buscar información sobre ocio, cultura y espectáculos y de los que sólo un $20 \%$ lee los medios tradicionales de prensa. Ello, unido a que el $60 \%$ de los españoles leen libros después de una recomendación personal, llevó a la biblioteca a una conclusión: el fomento de la lectura para los jóvenes podría hacerse por medio de sugerencias a través de un medio digital y participativo como es un blog bibliotecario.

En mayo de 2006 el blog de la Sedic lanzó la siguiente pregunta: ¿por qué hay tan pocas bibliotecas españolas que dispongan de este medio de difusión? Unas 600 en el mundo lo tenían por aquellas fechas, de las cuales eran españolas menos de 15, y de ellas sólo 2 universitarias, mientras que en EUA había más de 100.

Abrimos una cuenta en Blogspot para su alojamiento y comenzamos a publicar reseñas de novedades bibliográficas, invitando a la participación de los lectores. Pretendemos una actividad que fomente tanto la lectura como el aspecto social de la biblioteca. Mediante la exposición periódica de libros, por géneros, y comentados, nuestra intención es potenciar el uso de las colecciones de literatura e invitar a la colaboración en su selección. Se informa directamente a los usuarios sobre las novedades bibliográficas, se comentan las reseñas de los libros que se incorporan a la colección y es posible establecer una conversación sobre un libro determinado durante varios días. Además, crea una agenda cultural dinámica que sirve para anunciar actividades como presentaciones de nuevos títulos, foros sobre libros, conferencias de autores, relaciones entre el libro y el cine, cursos de verano o de humanidades basados en lecturas de narrativa, etc. "El aspecto social de las bibliotecas es probablemente mucho más importante de lo que nos imaginamos. Más que nunca, necesitamos lugares en los que la gente pueda reunirse. En las biblio- tecas del futuro podríamos tener lo que los franceses denominan cafésphilos, en los cuales la gente puede reunirse con filósofos, o salones literarios como los que existían en el siglo XVIII" (Berndton).

\section{Referencias bibliográficas}

Berndton, Maija. Soñando el futuro: ideas funky en la gestión de bibliotecas públicas. Consultado en: 19-12-06.

http://www.fundacionbertelsmann.org/bertelsmann-stiftung-de/ESP/media/ByG10.pdf

Candeira, Javier. La web como memoria organizada: el hipocampo colectivo de la red. Consultado en: 19-12-06.

http://biblioweb.sindominio.net/telematica/ para_can.htm

Celaya, Javier. Cómo fomentar la lectura y la escritura a través de los blogs. Consultado en: 19-12-06.

http://www.dosdoce.com/continguts/articulosOpinion/vistaSola_cas.php?ID $=80$

Leiva-Aguilera, Javier. "Breve aproximación a los blogs para unidades de información". En: Anuario ThinkEPI, 2007, pp. 166-168.

Francisco López Hernández, Honorio Penadés de la Cruz, Biblioteca de la Universidad Carlos III de Madrid.

pacol@db.uc3m.es

hpenades@db.uc3m.es 